\title{
Dimensions of the Ascending Aorta in Conotruncal Heart Defects
}

\author{
Amedeo Trippel · Sabine Pallivathukal • \\ Jean-Pierre Pfammatter · Damian Hutter · \\ Alexander Kadner $\cdot$ Mladen Pavlovic
}

Received: 3 September 2013 / Accepted: 2 January 2014/Published online: 28 January 2014

(C) Springer Science+Business Media New York 2014

\begin{abstract}
Dilatation of the ascending aorta is an important sequel in conotruncal anomalies, such as tetralogy of Fallot (TOF) or d-transposition of the great arteries (TGA). We measured dimensions and their progression at different levels of the ascending aorta in 80 patients. In TOF patients, mean $z$-score for aortic annulus was 1.65 (range -3.16-6.47), for sinus 1.93 (range $-2.28-5.39$ ), for stjunction 4.15 (range 0.0-8.18), and for ascending aorta 3.51 (range -1.23-6.36). Over time, annulus $z$-scores increased in the univariate analysis [0.07/year, $95 \%$ confidence interval (CI) $0.01-0.14 ; p=0.02]$, and this was unique to male patients $(0.08$ /year, $95 \%$ CI $0.00-0.15$; $p=0.05) . z$-scores of the ascending aorta decreased $(-0.1 /$ year, $95 \% \mathrm{CI}-0.18$ to $-0.02 ; p=0.02$ ), and this was confined to patients without aortic regurgitation (AR; $-0.09 /$ year, $95 \%$ CI -0.18 to $-0.01 ; p=0.04$ ). In TGA, mean $z$-score for the aortic annulus was 2.13 (range $-3.71-8.39$ ), for sinus 1.77 (range $-3.04-6.69$ ), for stjunction 1.01 (range -5.44-6.71), and for ascending aorta 0.82 (range -4.91-6.46). In bivariate analysis, annulus $z$ scores decreased in females $(-0.14 /$ year, $95 \% \mathrm{CI}-0.25$ to $-0.03 ; p=0.01)$ and in patients without AR $(-0.07 /$ year, $95 \% \mathrm{CI}-0.14-0.0 ; p=0.03)$. $z$-scores of the ascending
\end{abstract}

\footnotetext{
A. Trippel - S. Pallivathukal - J.-P. Pfammatter · D. Hutter · M. Pavlovic $(\bowtie)$

Department of Cardiology, University Hospital, 3010 Berne, Switzerland

e-mail: mladen.pavlovic@insel.ch
}

\author{
A. Trippel $\cdot$ S. Pallivathukal $\cdot$ J.-P. Pfammatter $\cdot$ D. Hutter \\ A. Kadner - M. Pavlovic \\ University Children's Hospital, 3010 Berne, Switzerland
}

\section{A. Kadner}

Department of Cardiovascular Surgery, University Hospital, 3010 Berne, Switzerland aorta increased significantly in males $(0.08 /$ year, $95 \% \mathrm{CI}$ 0.0 to $0.16 ; p=0.05)$ and in patients with $\mathrm{AR}(0.12 /$ year, $95 \%$ CI $0.03-0.21 ; p=0.01)$. In conclusion, TOF and TGA $z$-scores of the ascending aorta differ significantly from those of the normal population. Progression of $z$ scores over time is influenced by diagnosis, sex, and presence of AR.

Keywords Echocardiography - Dilatation - Ascending aorta $\cdot z$-Scores

\section{Background}

Conotruncal anomalies, such as tetralogy of Fallot (TOF) or transposition of the great arteries (TGA), are the most common severe congenital heart defects with incidences estimated at 421 (TOF) and 315 (TGA)/million live births [6]. Eventual complete surgical repair of these defects is generally performed during infancy with low early mortality and excellent late survival rates.

The growing consciousness of progressive aortic dilatation, which has been documented by accumulating numbers of published data $[7,11,19,20]$, can be attributed to improvements in cardiac imaging or to an increasing number of young patients advancing into adulthood. A recent shift of conceptualization is also changing from the paradigm of hemodynamic-poststenotic dilation [15] to a more important influence of intrinsic aortopathy [2, 14].

Unlike genetic disorders such as Marfan syndrome, the evolution and consequence of aortic dilation in patients with congenital cardiac defects remains less well defined. Although a portion of patients with congenital cardiac defects will go on to progress to aortic dilation and the accompanying complications of dissection or rupture, the 
risk factors for these complications have yet to be better understood. As a result of this uncertainty, questions regarding the requirement and best timing of eventual surgical or medical therapy have come to light [10].

\section{Objectives}

This study aimed to determine the frequency, rate of progression, and risk factors of aortic root and ascending aorta dilatation in patients with TOF and TGA. Furthermore, we sought to identify those patients with an increased risk for progressive dilatation or dissection to improve follow-up and eventual therapy.

\section{Methods}

The study was performed in accordance with the ethical standards laid down in the 1964 Declaration of Helsinki and its later amendments. Because it was a retrospective study using stored anonymous data, patients were not asked for informed consent.

\section{Patients}

Patients were selected through a review of the institutional cardiology database, which contains prospectively entered data on all patients seen by the staff cardiologists of the Division of Pediatric Cardiology at the University Children's Hospital, Berne, Switzerland.

The database, using two different diagnosis related codes - the International Classification of Disease2-10 code and the European Paediatric Cardiology Code (EPCC) code-was searched for patients listed under the following diagnoses: TOF/Fallot-type double-outlet right ventricle (TOF) and d-transposition of the great arteries (TGA). All echocardiograms had been saved on VHS cassette if recorded before 2008 or on a digital database if recorded in 2008 or later. All patients with d-TGA underwent arterial switch operation (ASO) during the neonatal period and had no associated cardiac malformations, such as ventricular septal defect (VSD) or forestation. In TOF patients, there were none with pulmonary artesia apart from one patient who received a neonatal modified BlalockTaussig shunt; corrective surgery was performed when patients were between 6 and 9 months of age.

The echocardiograms of all patients were reviewed for aortic dimensions. The respective reports were reviewed for clinical data: height, weight, age, sex, surgeries undergone, and presence of aortic stenos is (AS) or regurgitation (AR).
Table 1 Variables recorded for each echocardiogram

Height
Weight
Diagnosis group
Sex
Age
Aortic annulus diameter
Aortic sinus diameter
Sinotubular junction diameter
Ascending aorta diameter at the level of the right pulmonary artery
Surgeries undergone
AR
AS

Children were included if they satisfied the following criteria: $<18$ years of age at the time of first echocardiography; echocardiographic confirmation of one of the abovementioned diagnoses by a staff pediatric cardiologist; at least three technically adequate two-dimensional echocardiograms during the last 15 years with each one at least 1 year apart; and concomitant height and weight measurements available for the recorded echocardiograms.

Exclusion criteria were poor quality of the echocardiography images (insufficient to measure all aortic diameters), bicuspid aortic valve, or death during follow-up.

Three hundred forty-three patients with one of the above-mentioned diagnoses were identified. Of these, 82 fulfilled the inclusion criteria and were divided into two diagnosis-related groups: TOF (TOF and Fallot-type DORV patients) and TGA. In these 82 patients, a total of 310 echocardiograms with technically adequate measurable images were included in the study.

\section{Variables}

All patients were assigned to a diagnosis group, and their height, weight, sex, and age was noted. The number of surgeries undergone was also included in the variables together with the presence or absence of AR or AS. Further variables were the four aortic diameters at the levels of the annulus, aortic sinus, sinotubular $(\mathrm{s}-\mathrm{t})$ junction, and ascending aorta (Table 1).

\section{Measurements}

Transthoracic echocardiography was performed using an Acuson Sequoia 512 (Siemens Medical Solutions, Mountain View, CA). Patients were placed in either the left lateral position or a supine position under rest conditions. The aortic root diameters were measured in two-dimensional mode at four levels, i.e., aortic annulus, sinuses of 


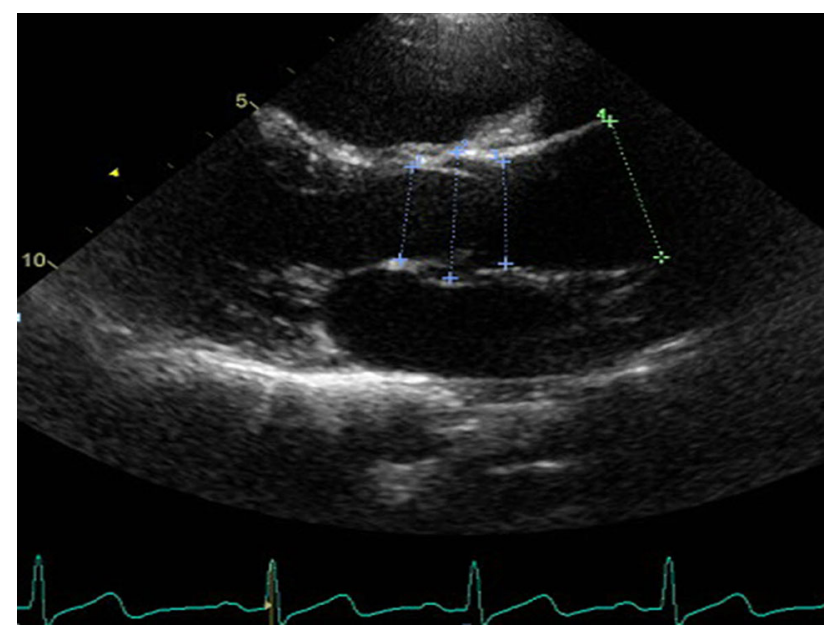

Fig. 1 Long-axis view of ascending aorta. Four measurement levels: Aortic annulus (1), sinus of Valsalva (2), sinotubular junction (3), ascending aorta (4)

Valsalva (sinus), st-junction, and ascending aorta (Fig. 1) during systole and using the leading technique according to the latest 2005 American Society of Echocardiography (ASE) chamber-quantification guidelines [8]. The best parasternal long-axis view was used. Appropriately magnified views were used for greater precision.

Echocardiographic images saved in the digital database were measured offline with Syngo Dynamics Software (Siemens AG, Erlangen, Germany); the images saved on VHS were measured on a Sony Trinitron television based on the calibration markers as reference.

The offline measurements were made by 2 medical students in their fourth year of studies, and 20 casual measurements for each level were compared with those made by the pediatric cardiologist to determine interobserver reliability using the statistical method proposed by Bland and Altman [1].

\section{Definition of Aortic Root Dilatation}

Because aortic dimensions depend on body surface area (BSA) and sex [17], $z$-scores were calculated using the equations proposed by Gautier et al. [4] with $\mathrm{z}$ defined as the number of SDs greater or less than the predicted mean diameter. An aortic diameter was considered to be dilated when the $z$-score was $\geq 2$ with a specificity of $97.7 \%$.

BSA used in the $z$-score formulas was computed using the Haycock formula [5]: BSA $\left(\mathrm{m}^{2}\right)=$ weight $(\mathrm{kg})^{0.5378} \times$ height $(\mathrm{cm})^{0.3964} \times 0.024265$.

\section{Statistical Analysis}

Statistical analysis was performed using SPSS software (SPSS, Chicago, IL) employing linear mixed-effects in univariate and bivariate analyses for each level. A $p$ value $\leq 0.05$ was considered significant.

\section{Results}

Three hundred eight measurements were recorded in 80 patients, of which 150 were from 42 TOF patients. Mean age at the time of measurements was 12.6 years (range 0.4-24.0) for TOF patients and 9.0 years (range 0.0-17.6) for TGA patients. The excluded patients were not different from the studied patients regarding age and other demographic variables.

There was a predominance of male patients in both diagnosis groups with only 40 of 150 measurements in girls with TOF and 31 of 158 measurements in girls with TGA, respectively (Table 2). No deaths were recorded. Because age at repair was homogenous in TOF as well as in TGA patients, this was not taken into account in the statistical analysis.

\section{TOF Patients}

Of the entire group, 149 measurements were from previously operated patients, and only 1 measurement was made before surgery.

\section{Aortic valve function}

No AR was found in the majority of the measurements $(n=114)$. Mild aortic valve regurgitation was identified in 34 measurements, and in only 2 images was moderate aortic insufficiency noted. In none of the analyzed echocardiographic images was stenosis of the aortic valve was found.

Table 2 Patient characteristics

\begin{tabular}{lll}
\hline Characteristics & TOF & TGA \\
\hline Age in years (range) & $12.6(0.4-24.0)$ & $9.0(0.0-17.6)$ \\
Sex (M:F) & $110: 40$ & $127: 31$ \\
No AR & 114 & 72 \\
No. of surgeries $(n)$ & $149 / 150$ & $158 / 158$ \\
\hline
\end{tabular}

Table 3 Mean $z$-scores (overall)

\begin{tabular}{lcc}
\hline Level & TOF & TGA \\
\hline Annulus & 1.65 & 2.13 \\
Sinus & 1.93 & 1.77 \\
ST junction & 4.15 & 1.01 \\
Ascending aorta & 3.51 & 0.82 \\
\hline
\end{tabular}


Table 4 Mean $z$-scores: initial and last

\begin{tabular}{lll}
\hline Level & TOF & TGA \\
\hline Annulus: initial & 0.06 & 2.3 \\
Annulus: last & 1.54 & 2.14 \\
Sinus: initial & 1.64 & 2.2 \\
Sinus: last & 1.89 & 1.52 \\
ST junction: initial & 3.86 & 0.95 \\
ST junction: last & 4.22 & 1.29 \\
Ascending aorta: initial & 4.95 & 0.4 \\
Ascending aorta: last & 2.99 & 1.3 \\
\hline
\end{tabular}

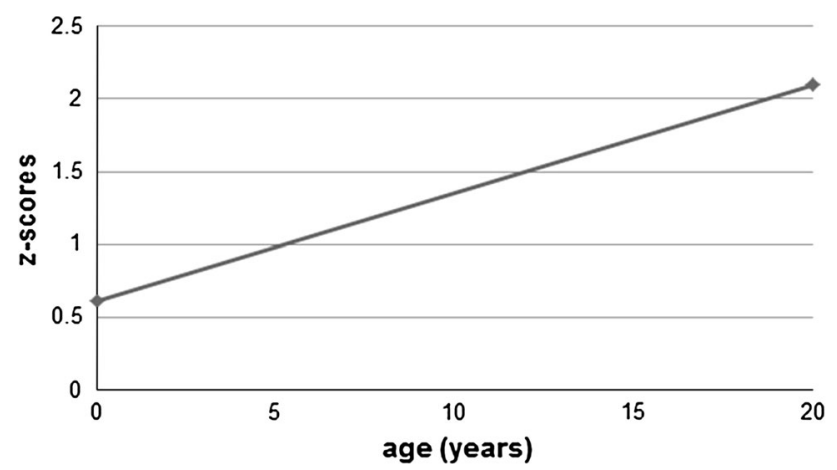

Fig. 2 Annulus $z$-scores increasing in TOF patients significantly over time $(p=0.02)$

Table 5 Change of $z$-scores over time in TOF patients

\begin{tabular}{lll}
\hline Level & Change/year (range) & $p$ \\
\hline Annulus & $0.07(0.01-0.14)$ & 0.02 \\
Sinus & $0.01(-0.04-0.07)$ & 0.65 \\
ST junction & $0.02(-0.04-0.07)$ & 0.5 \\
Ascending aorta & $-0.1(-0.18-0.02)$ & 0.02 \\
\hline
\end{tabular}

\section{Mean z-Scores}

Overall, mean $z$-score for aortic annulus in the TOF group was 1.65 (range -3.16-6.47), for sinus 1.93 (range -2.28-5.39), for st-junction 4.15 (range 0.0-8.18), and for ascending aorta 3.51 (range $-1.23-6.36$, Tables 3,4 ). The overall percentage of patients with $z$-score $>2$ at the different parts of the aorta in TOF patients was $40 \%$ at the annulus, $48 \%$ at the sinus, $93 \%$ at the st-junction, and $88 \%$ at the ascending aorta.

\section{Longitudinal analysis}

\section{Aortic annulus}

In the univariate analysis, overall annulus $z$-scores increased significantly over time (0.07/year, $95 \%$ CI

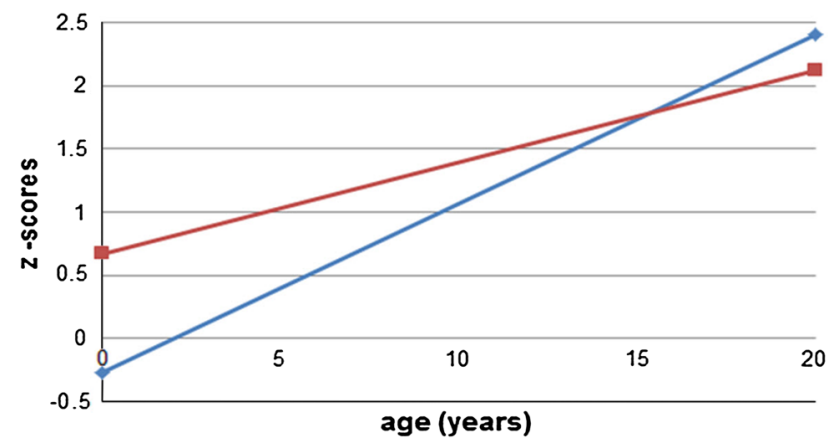

Fig. 3 Increase of $z$-scores of aortic annulus in TOF patients over time regardless of presence (blue, $p=0.01$ ) or absence (red, $p=0.03)$ of $\mathrm{AR}$

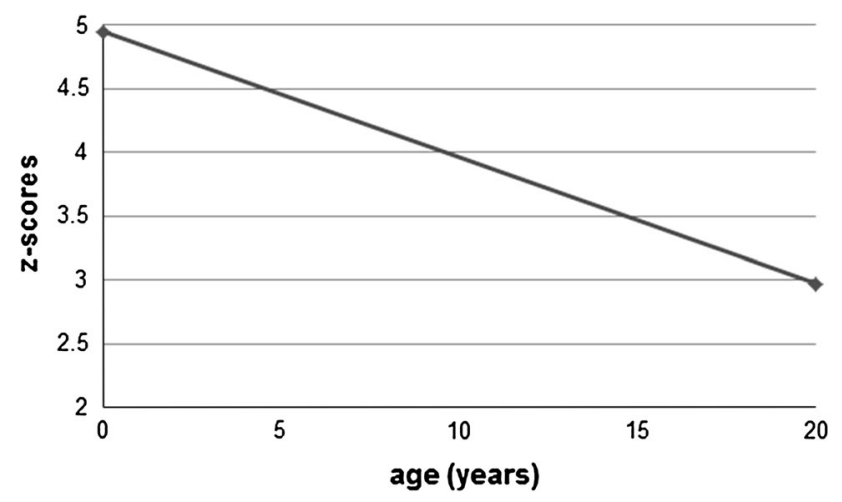

Fig. $4 z$-scores of ascending aorta in TOF patients decreasing significantly $(p=0.02)$ with a high starting point (intercept 4.95 , $p<0.01)$

0.01-0.14; $p=0.02$, Fig. 2; Table 5). In the bivariate analysis, only male patients had a significant increase of $z$ score of $0.08 /$ year (95 \% CI $0.00-0.15 ; p=0.05$ ), whereas in females the increase was not significant (0.09/year, $95 \%$ CI -0.04-0.22; $p>0.15)$. Patients had a significant increase of $z$-scores over time regardless of presence $(0.13 /$ year, $95 \%$ CI $0.03-0.24 ; p=0.01)$ or absence $(0.07 /$ year, $95 \%$ CI $0.01-0.14 ; p=0.03$ ) of AR (Fig. 3).

\section{Sinus of Valsalva}

At the level of the aortic sinus, no significant change of $z$-scores could be determined neither in the univariate $(0.01 /$ year, $95 \%$ CI $-0.04-0.07 ; p>0.5)$ nor in the bivariate analysis comparing males with females [0.01/year, $95 \% \mathrm{CI}-0.06-0.07$ $(p>0.5)$ vs. $0.04 /$ year, $95 \% \mathrm{CI}-0.07-0.15(p>0.4)]$ or presence and absence of AR [0.0/year, $95 \%$ CI $-0.10-0.10$ $(p>0.5)$ vs. $0.02 /$ year, $95 \%$ CI $-0.04-0.07(p>0.5)]$.

\section{Sinotubular Junction}

No significant trend regarding $z$-scores of the st-junction could be identified in univariate (0.02/year, $95 \%$ CI 


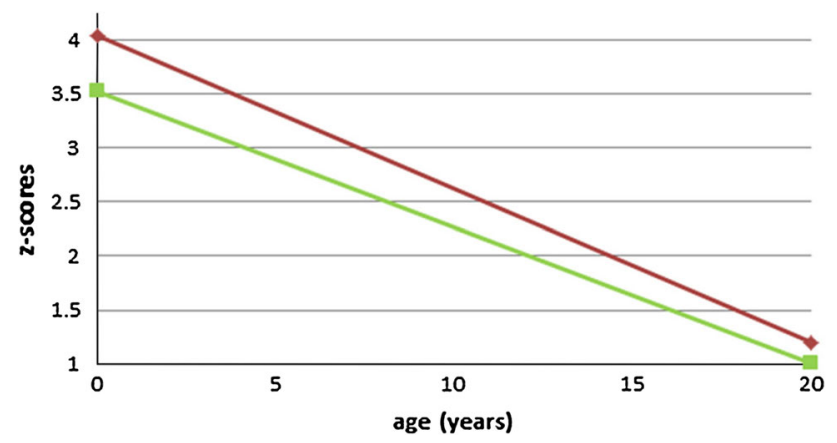

Fig. $5 z$-scores of annulus (red) and sinus (green) in the female TGA patients decreasing significantly over time (each $p=0.01$ )

$-0.04-0.07 ; p>0.5$ ) or bivariate analysis in the comparison of female with male patients $[-0.01 /$ year, $95 \% \mathrm{CI}$ $-0.12-0.10(p>0.5)$ vs. 0.03/year, $95 \%$ CI $-0.04-0.09$ $(p=0.37)]$ or in the insufficiency of the aortic valve $[-0.06 /$ year, $95 \% \mathrm{CI}-0.16-0.04(p=0.25)$ in AR vs. $0.02 /$ year, $95 \% \mathrm{CI}-0.03-0.08(p=0.39)$ without AR].

\section{Ascending Aorta}

In the univariate analysis, $z$-scores of the ascending aorta decreased significantly $(-0.1 /$ year, $95 \% \mathrm{CI}-0.18$ to $-0.02 ; p=0.02$ ) with a high starting point (intercept 4.95 , $p<0.01$ ) (Fig. 4). In the bivariate analysis, this decrease was confined to patients without AR ( -0.09 /year, $95 \%$ CI -0.18 to $-0.01 ; p=0.04)$. No significant decrease was found in the subgroups of male or female patients $[-0.1 /$ year, $95 \%$ CI $-0.20-0.01(p=0.07)$ vs. $-0.09 /$ year, $95 \% \mathrm{CI}-0.23-0.06(p>0.2)]$ or in patients with $\mathrm{AR}$ $(-0.1 /$ year, $95 \% \mathrm{CI}-0.25-0.04 ; p>0.1)$.

\section{TGA Patients}

All measurements in patients of the TGA group included in this study were made after surgery. One hundred twentynine measurements were made in patients operated once, 16 in patients reoperated once, 9 in patients reoperated twice, and 4 in patients reoperated thrice.

\section{Aortic Valve Function}

In approximately half of the observations, no regurgitation was identified, namely, in 72 cases. Mild regurgitation was found in 78 measurements, and the remaining 8 cases presented moderate insufficiency. Of the 158 measurements that were performed and included in this study, in only 1 case was mild AS was found.
Table 6 Change of $z$-scores over time in TGA patients

\begin{tabular}{lll}
\hline Level & Change/year (range) & $p$ \\
\hline Annulus & $-0.01(-0.06-0.05)$ & 0.8 \\
Sinus & $-0.03(-0.08-0.01)$ & 0.2 \\
ST junction & $0.02(-0.04-0.08)$ & 0.6 \\
Ascending aorta & $0.04(-0.02-0.11)$ & 0.2 \\
\hline
\end{tabular}

Mean z-Scores

Overall, mean $z$-score for aortic annulus in the TGA group was 2.13 (range -3.71-8.39), for sinus 1.77 (range -3.04-6.69), for st-junction 1.01 (range -5.44-6.71), and for ascending aorta 0.82 (range $-4.91-6.46$; Table 3). In the TGA group, the percentage of patients with $z$-score $>2$ at the different parts of the aorta was $62 \%$ at the annulus, $59 \%$ at the sinus, $41 \%$ at the st-junction, and $28 \%$ at the ascending aorta, respectively.

Longitudinal Analysis

\section{Aortic Annulus}

In univariate analysis, no significant change of $z$-scores was found $(-0.01 /$ year. $95 \% \mathrm{CI}-0.06-0.05 ; p>0.5)$. In the bivariate analysis, $z$-scores in female patients decreased significantly over time [ $-0.14 /$ year, $95 \%$ CI -0.25 to -0.03 $(p=0.01)$; Fig. 5; Table 6], whereas in male patients $(0.03$ / year, $95 \% \mathrm{CI}-0.03-0.1 ; p>0.2)$, no change was observed. The presence or absence of regurgitation did not influence the size of the aortic annulus in the studied TGA population [0.04/ year, $95 \% \mathrm{CI}-0.4-0.12(p=0.18)$ for AR vs. $-0.05 /$ year, $95 \% \mathrm{CI}-0.13-0.03(p>0.3)$ without AR].

\section{Sinus of Valsalva}

In the univariate analysis, no significant data were identified $(-0.03 /$ year, $95 \% \mathrm{CI}-0.08-0.01 ; p=0.16)$, whereas in bivariate analysis compared with male patients $(0.0 /$ year, $95 \%$ CI $-0.06-0.5 ; \quad p>0.5), \quad z$-scores in females decreased significantly over time [-0.13/year, $95 \%$ CI -0.22 to $-0.03(p=0.01)$; Fig. 5]. Patients without AR showed a decrease of $z$-scores over time $(-0.07 /$ year, $95 \%$ CI $-0.14-0.0 ; p=0.03)$, whereas patients with AR showed no significant change (0.0/year, $95 \%$ CI $-0.07-0.07 ; p>0.5$ ).

\section{Sinotubular Junction}

At the level of the st-junction, no significant change was found, neither in the univariate $(0.02 /$ year, $95 \%$ CI $-0.04-0.08 ; p>0.5)$ nor the bivariate analysis $[-0.07 /$ 
year, $95 \%$ CI $-0.20-0.04(p=0.2)$ for female vs. 0.04/ year, $95 \%$ CI $-0.02-0.12(p=0.17)$ for males] [0.07/ year, $95 \%$ CI $-0.01-0.16(p=0.07)$ with AR vs. $-0.04 /$ year, $95 \% \mathrm{CI}-0.12-0.04(p=0.32)$ without AR].

\section{Ascending Aorta}

In the univariate analysis, no significant data were identified $(0.04$ year. $95 \% \mathrm{CI}-0.02-0.11 ; p=0.17)$, whereas in the bivariate analysis $z$-scores in measurements of male patients increased significantly over time $(0.08 /$ year, $95 \%$ CI $0.0-0.16 ; p=0.05)$; however, this was not true in female patients $(-0.05 /$ year, $95 \%$ CI $-0.18-0.08$; $p=0.5)$. Moreover, AR had a significant influence on progression of $z$-scores over time $(0.12 /$ year, $95 \% \mathrm{CI}$ $0.03-0.21 ; p=0.01)$, whereas patients without AR showed no significant change $(-0.04 /$ year, $95 \%$ CI $-0.13-0.06 ; p=0.4)$.

Interobserver Reliability

Analysis of interobserver reliability performed on data from 20 randomly chosen patients for all four levels showed overall excellent agreement. For the annulus, the mean bias (mean of differences) was 0.3 for the first student and 0.15 for the second; the limits of agreement (mean $\pm 1.96 \mathrm{SD}$ ) were -0.82 and 1.42 (respectively -1.68 and 1.98). For the aortic sinus, the mean biases were -0.1 and 0.05 , and the limits of agreement were -1.36 , 1.16 and $-2.37,2.47$. At the level of the st-junction, the mean biases were 0.55 and 0.2 , whereas the limits of agreement were $-0.64,1.74$ and $-1.87,2.27$. Finally for the ascending aorta, the mean biases were 0 and -0.5 , and the limits of agreement $-1.80,1.80$ and $-2.24,1.24$.

\section{Discussion}

The study population of TOF and TGA patients did not differ regarding age and sex distribution. Moreover, almost all measurements were performed in postoperative subjects. Normal function of the aortic valve was found in nearly two-thirds of the TOF patients and in half of the TGA patients; the majority of the remainder had only mild valvar dysfunction without hemodynamic consequences and need for reoperation.

Our study showed that patients with conotruncal anomalies (TOF and TGA) have $z$-scores of the ascending aorta that are significantly different from the normal population. The two patient groups differed regarding the extent of dilatation of the different sites of the ascending aorta. Moreover, in our patients, specific parts of the ascending aorta showed different modes of change of $z$ - scores over time. This was influenced by diagnosis, sex, and presence of AR.

Former articles have underlined the importance of a better understanding of the evolution of patients with conotruncal anomalies. So did a study by Niwa et al. [11], which identified a subset of adult TOF patients exhibiting ongoing aortic root dilatation late after repair, as in our study.

In our study, TOF patients had increased $z$-scores of the distal ascending aorta (st-junction and ascending), whereas Chong et al. found a high prevalence of aortic dilation at all levels of the ascending aorta in children after corrective surgery for TOF [3]. In these patients, the annulus $z$-scores progressed over time. This was confined to male patients regardless of the presence or absence of aortic valve regurgitation. The ascending aorta of these patients, although having increased $z$-scores overall, displayed even a decrease of $z$-scores over time; however, this was true only in those patients without regurgitation of the aortic valve. In contrast to our study, the only article analyzing the change of $z$-scores over time in TOF patients showed increasing $z$-scores especially at the aortic annulus level [3].

In TGA patients primarily, the annulus tended to larger dimensions. This is in line with former reports [16] showing that diameters of aortic root 20 years after ASO were significantly wider at the proximal levels of the ascending aorta (annulus and sinus) compared with a normal population, although they were less wide (but still significant) at distal levels of the ascending aorta.

The $z$-scores of the aortic annulus of the entire TGA group did not change significantly. AR had no influence on this finding. However, in female TGA patients, $z$-scores decreased significantly over time. The same was true for $z$ scores of the sinus of Valsalva, which decreased only in females but not in males. Moreover, in this region regression of $z$-scores was found only in patients without AR. The $z$-scores of the ascending aorta progressed over time; however, they did so only in males and those patients with AR. Our results cannot be compared with those of a previous article [13] identifying previous pulmonary artery banding (PAB) as an independent risk factor for the development of aortic root dilation because in our group patients with additional VSD or PAB were excluded.

We found a significant influence of sex on the susceptibility to dilation of the ascending aorta. Male TOF and TGA patients were more prone to greater rates of dilation compared with females. The sex influence was noticed either as a greater increase of $z$-scores in males or a greater decrease of $z$-scores in female patients, which is in line with former reports $[11,20]$.

The relation between AR and dilation of the ascending aorta in our patients suggests that regurgitation itself is a 
hemodynamic risk factor for ongoing dilation. This is in line with data from Rutz et al. [12] showing impaired distensibility with a subsequent dilation in TGA patients only at the level of the ascending aorta. Although AR as a hemodynamic risk factor modulated the evolution of dilation, there is accumulating evidence that an intrinsic aortopathy in conotruncal anomalies is part of the problem [2,14]. Yetman and Graham [20] highlighted some similarities in pathophysiologic alteration between conotruncal defects and Marfan syndrome. TGF $\beta-1$ has been discussed as a possible modulator of ongoing dilation development. Defective TGF $\beta-1$ receptor Alk5 is known to play a key role in severe outflow-tract defects [18]. The recent evidence linking Marfan syndrome to conotruncal defects might bring to a new concept of managing aortic dilation by observing circulating TGF $\beta-1$ levels over time and eventually even antagonizing it as suggested by Matt et al. [9].

In contrast to TGA patients with previous $\mathrm{PAB}$ and associated malformations, such as VSD or coarctation, no significant progression of aortic dimensions was found in our patients. However, those patients with mild to moderate $\mathrm{AR}$ and male sex had ongoing dilation. Therefore, we believe that "simple TGA" patients are at lower risk for a significant course of aortic dilation, whereas this might not be true for the subgroup with male sex and aortic valve dysfunction.

Magnetic resonance imaging and computed tomography scans have significantly improved the delineation of anatomy, but for standard surveillance echocardiography should be adequate. To date our results will not change the current management of surveillance of these patients. If $z$-score is increased but remains stable, patients will be followed-up annually or every second year. However, progression has been shown to occur in male patients and those with valve dysfunction; this subgroup in particular might benefit from treatment with afterload-lowering medication. Additional research, prospective studies, and long-term follow-up are needed for patients with conotruncal defects to detect "cutoff" values for surgical intervention and to prove if there is a true benefit from an early drug therapy.

\section{References}

1. Bland JM, Altman DG (1986) Statistical methods for assessing agreement between two methods of clinical measurement. Lancet 1(8764):307-310

2. Boyum J, Fellinger EK, Schmoker JD et al (2004) Matrix metalloproteinase activity in thoracic aortic aneurysms associated with bicuspid and tricuspid aortic valves. $\mathbf{J}$ Thorac Cardiovasc Surg 127:686-691
3. Chong WY, Wong WH, Chiu CS, Cheung YF (2006) Aortic root dilation and aortic elastic properties in children after repair of tetralogy of Fallot. Am J Cardiol 97:905-909

4. Gautier M, Detaint D, Fermanian C et al (2010) Nomograms for aortic root diameters in children using two-dimensional echocardiography. Am J Cardiol 105:888-894

5. Haycock GB, Schwartz GJ, Wisotsky DH (1978) Geometric method for measuring body surface area: a height-weight formula validated in infants, children, and adults. J Pediatr 93:62-66

6. Hoffman JI, Kaplan S (2002) The incidence of congenital heart disease. J Am Coll Cardiol 39:1890-1900

7. Holman E (1954) The obscure physiology of poststenotic dilatation; Its relation to the development of aneurysms. J Thorac Surg 28:109-133

8. Lang RM, Bierig M, Devereux RB et al (2005) Recommendations for chamber quantification: a report from the American society of echocardiography's guidelines and standards committee and the chamber quantification writing group, developed in conjunction with the European association of echocardiograph. J Am Soc Echocardiogr 18:1440-1463

9. Matt P, Schoenhoff F, Habashi J et al (2011) Circulating TGF- $\beta$ in Marfan's syndrome. Circulation 120:526-532

10. McMahon CJ, Ravekes WJ, Smith EO et al (2004) Risk factors for neo-aortic root enlargement and aortic regurgitation following arterial switch operation. Pediatr Cardiol 25:329-335

11. Niwa K, Siu SC, Webb GD, Gatzoulis MA (2002) Progressive aortic root dilatation in adults late after repair of tetralogy of Fallot. Circulation 106:1310-1311

12. Rutz T, Max F, Wahl A, Wustmann K, Khattab K, Pfammatter JP, Kadner A et al (2012) Distensibility and diameter of ascending aorta assessed by cardiac magnetic resonance imaging in adults with tetralogy of Fallot or complete transposition. Am J Cardiol 110:103-108

13. Schwartz ML, Gauvreau K, del Nido P, Mayer JE, Colan SD (2004) Long-term predictors of aortic root dilation and aortic regurgitation after arterial switch operation. Circulation 14:II128-II132

14. Sundt TM 3rd (2010) Replacement of the ascending aorta in bicuspid aortic valve disease: where do we draw the line? J Thorac Cardiovasc Surg 140:41-51

15. Tan JL, Davlouros PA, McCarthy KP, Gatzoulis MA, Ho SY (2005) Intrinsic histological abnormalities of aortic root and ascending aorta in tetralogy of Fallot: evidence of causative mechanism for aortic dilatation and aortopathy. Circulation 112:961-968

16. Vandekerckhove KD, Blom NA, Lalezari S, Koolbergen DR, Rijlaarsdam ME, Hazekamp MG (2009) Long-term follow-up of arterial switch operation with an emphasis on function and dimensions of left ventricle and aorta. Eur J Cardiothorac Surg 35:582-587

17. Vasan RS, Larson MG, Levy D (1995) Determinants of echocardiographic aortic root size. The framingham heart study. Circulation 91:734-740

18. Wang J, Nagy A, Larsson J, Dudas M, Sucov HM, Kaartinen V (2006) Defective ALK5 signaling in the neural crest leads to increased postmigratory neural crest cell apoptosis and severe outflow tract defects. BMC Dev Biol 6:51

19. Warren E, Boyd ML, O'Connell C, Dodds L (2006) Dilatation of the ascending aorta in paediatric patients with bicuspid aortic valve: frequency, rate of progression and risk factors. Heart 92:1496-1500

20. Yetman AT, Graham T (2009) The dilated aorta in patients with congenital cardiac defects. J Am Coll Cardiol 53:461-467 